\title{
The hydrophobicity of surfaces with micro-structures
}

\author{
Xu Zheng and Zhanhua Silber-Li* \\ The State Key Laboratory of Nonlinear Mechanics, Institute of Mechanics, Chinese Academy of Sciences
}

\begin{abstract}
Micro/nano structures on the surface of the superhydrophobic lotus leaves have been observed with Scanning Electron Microscope (SEM) images, and the hydrophobicity enhanced by rough structures on hydrophobic surface has attracted lots of interests. This paper studied on the effects of micro-structures. From replication of lotus leaves surface by using poly-dimethylsiloxane (PDMS), micro-structures can be obtained on the artificial surface. The contact angle (CA) on this surface is about $140^{\circ}$, which means the microstructures make important efforts. Considering the characteristics of Cassie's law and Wenzel's law, this paper proposes a revised formula to describe the relations between contact angles and geometric sizes of surface micro-structures. An optimum value of $b / a$ (a is the width of the micro-structures, $b$ is the interspace between microstructures) is given for maximum $\mathrm{CA}$.
\end{abstract}

Keywords-Lotus leaf, micro-/nano-structure, contact angle, (super) hydrophobicity,PDMS

\section{INTRODUCTION}

Different kinds of methods have been tried to achieve hydrophobic or super hydrophobic surfaces, and to apply these surfaces to practical uses. Chemical methods, such as low surface energy coating [1], are convenient, but the maximum contact angle on a flat surface with attainable lowest free energy coating is only $115.2^{\circ}$, as was pointed out by Nakajima [2]. Since Barthlott and Neinhuis [3] discovered that the selfcleaning effect of lotus leaves is caused by the roughness of their surfaces, Bechert et al. [4], Furstner et al. [5], Barthlott and Neinhuis [6] have shown that there are many kinds of rough structures on some hydrophobic leaf surfaces. It's the inspiration from nature.

In this paper, with SEM (FEI Sirion 400NC), we have observed that, there are micro/nano double-layer structures on the surfaces of lotus leaves. Many "small protrusions" are distributed on the surfaces, about $7 \mu \mathrm{m}$ in width, $10 \mu \mathrm{m}$ in height and $9 \mu \mathrm{m}$ in distance (Fig. 1a). After being magnified to about $15900 \times$, SEM picture shows that there are nanostructures upon the "small protrusions" and among the "small protrusions" on the leaf surface (Fig. 1b). Further magnification reveals "deer horn-like" nano-structures upon the "small protrusions" (Fig. 1c and Fig. 1d), and "branchlike" nano-structures among the "small protrusions" (Fig. 1e and Fig. 1f). These nano-structures are about 80-100 nanometer in diameter. With image processing, we estimated that the interspace proportions of the nano-structures upon the "small protrusions" and among the "small protrusions" are about $15 \%$ and $55 \%$, respectively (Fig. 1c $\sim$ Fig. 1f). The CA of lotus leaves is measured as $157^{\circ}$ (Fig. 2), so it is obvious that super hydrophobicity of lotus leaves surface is related with these micro/nano structures.

This project was funded by Major Innovation Project (KJCX2-SW-L2) of Chinese Academy of Science and National Natural Science Foundation of China (10272107).

*Contact author:lili@imech.ac.cn

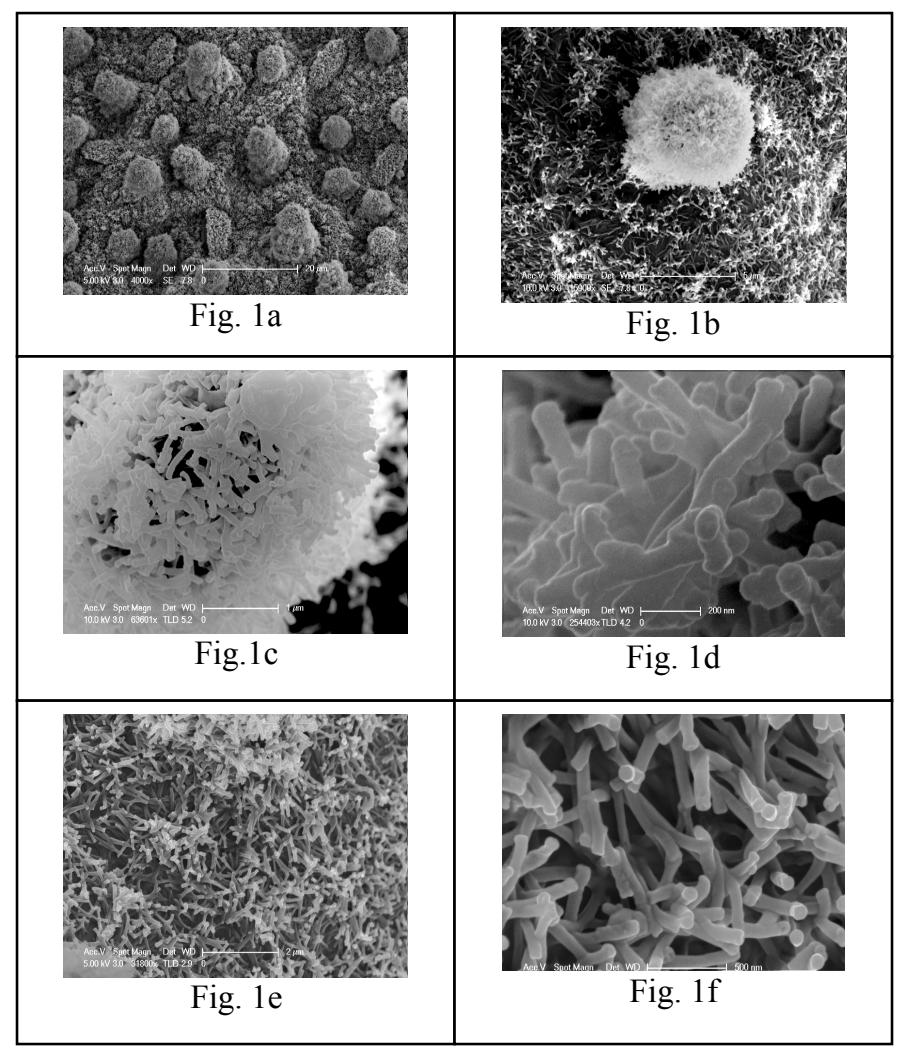

Figure 1. Micro/nano structures on lotus leaf surfaces.

The hydrophobicity resulting from nano-structures has also been reported recently. Gao and Jiang [7] discovered the nanostructures of water strider's water-repellent legs. Lee et al. [8] studied the hydrophobic effect of aligned nano-structures on Cicada orni's wings. Watson and Watson [9] described some interesting natural nano-structures on insects.

The effects of rough structures are obvious, but the mechanism still needs to be studied. Öner and McCarthy [10] tried to study the characteristic length scale of the rough structures. Onda et al. [11] tried to use fractal theory to explain the mechanism. Patankar [12] proposed a double-layer model. To understand the hydrophobicity of multiple structures, it is advisable to study micro or nano structures separately. This paper focuses on the hydrophobicity of micro-structures on lotus leaves. We replicated lotus leaves surfaces by PDMS, which reserved the micro-structures of lotus leaves surfaces well, then measured the surface CA of PDMS replica and the geometric sizes of the micro-structures (section II), analyzed the relation between geometric sizes of surface microstructures and CA, and proposed a revised formula to describe the relations between $\mathrm{CA}$ and geometric sizes of surface micro-structures (section III) 


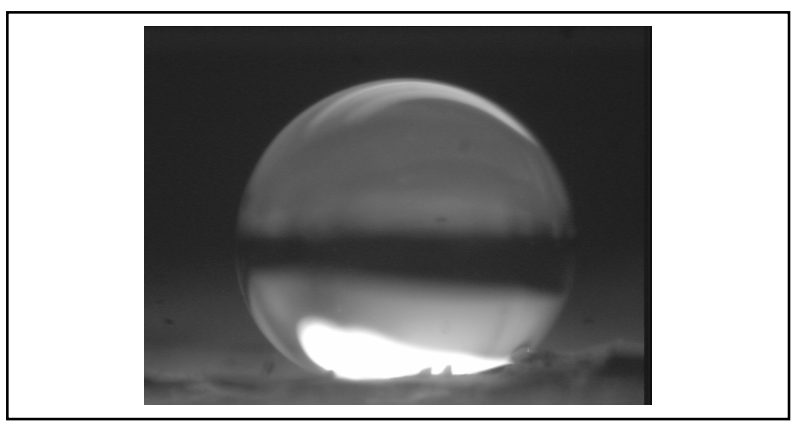

Figure 2. Measurement of CA on lotus leaf, $\mathrm{CA}=157^{\circ}$.

\section{EXPERIMENTS}

\section{A. Replicating lotus leaves by PDMS}

PDMS (poly-dimethylsiloxane) is a transparent liquid in normal temperature and normal pressure. Mixed with ten parts of base material and one part curing agent, and cured under a special temperature, PDMS will form solid hydrophobic surface, whose CA is about $100^{\circ}$. McDonald and Whitesides [13] gave a description PDMS's characteristics and its applications in MEMS. In our experiments, PDMS was used to cover a piece of lotus leaf directly. The first pattern was obtained while PDMS was separated from the lotus leaf (Fig. 3 step 1). Then PDMS was covered on the first pattern, and separated again to obtain the second pattern. The second pattern was the replica of the lotus leaf (Fig. 3 step 2). So this method provides us a convenient way to obtain hydrophobic lotus leaf like surface.

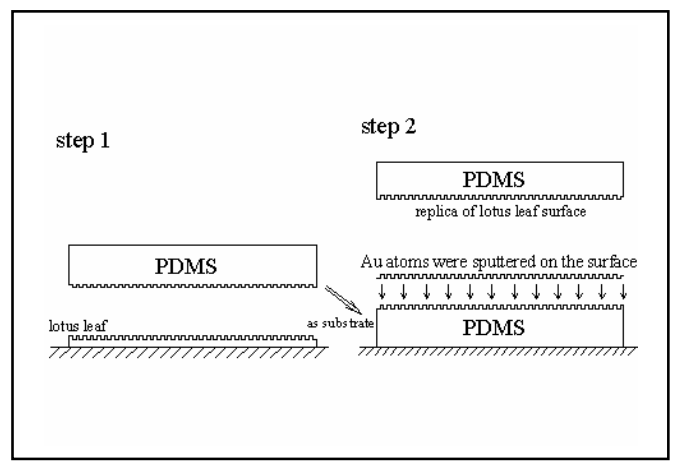

Figure 3. Schematic diagram of the lotus leaf replication process with PDMS.

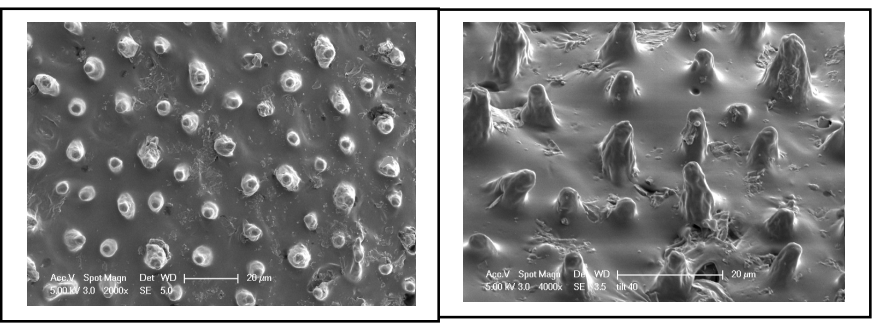

Figure 4. SEM pictures of PDMS replica sample 1.
TABLE I. THE GEOMETRIC SIZES OF MICRO-STRUCTURES OF LOTUS LEAVES AND PDMS REPLICAS, AND THE CA MEASUREMENT RESULTS.

\begin{tabular}{|c|c|c|c|l|}
\hline & $a(\mu \mathrm{m})$ & $\mathrm{h}(\mu \mathrm{m})$ & $\mathrm{b}(\mu \mathrm{m})$ & $\mathrm{CA}$ \\
\hline PDMS sample 1 & 3 & 9 & 9 & $146^{\circ}$ \\
\hline PDMS sample 2 & 4 & 7 & 9 & $138^{\circ}$ \\
\hline PDMS sample 3 & 5 & 5 & 9 & $130^{\circ}$ \\
\hline Lotus leaf & 7 & 10 & 9 & $157^{\circ}$ \\
\hline
\end{tabular}

\section{B. Geometric sizes of surface structures}

Fig. 4a and Fig. 4b show the surface micro-structures of the replica (Sample 1). The definitions of geometric sizes of micro-structures are given in Fig.5. We measured the geometric sizes of micro-structures of lotus leaves and PDMS replicas by SEM pictures, the results are shown in Table.1. Because the proportion of base material to curing agent was not 10:1 exactly, and the curing time and curing temperature were different in the replication processes, the surface geometric sizes of different samples were not exactly same, but $b$ is same in 3 cases.

\section{Measuring the contact angle}

A water droplet was gently put on rough surfaces for measuring CA. The diameter of droplets in the experiment was about 1.2 millimeter, the Bond number was about 0.05 , so we could neglect the effect of gravity in the experiment. The contact angles of three samples of PDMS replicas were $146^{\circ}$, $138^{\circ}$ and $130^{\circ}$, respectively (Fig. 6 shows the CA of the replica in sample 1). Because of the difficulty of separating between these two patterns, we had to sputter (BAL-TEC $\mathrm{SCD} 050$ ) a layer of $\mathrm{Au}$ atoms (about 5nm in thickness) as separating layer. In order to know the effect of $\mathrm{Au}$ atoms as a separating layer in the PDMS replica process before and after using Au coating, we measured the contact angles of PDMS flat surface. The CA of the PDMS flat surface decreased from $104^{\circ}$ to $96^{\circ}$ after using a separating layer of $\mathrm{Au}$, therefore the CA values in Tablel are underestimated.

\section{ANALYSIS AND DISCUSSION}

\section{A. Theoretical background}

Contact angle is an important parameter of hydrophobicity. According to Young's law, the contact angle $\theta$ in a flat, uniform and isotropic surface is expressed as:

$\cos \theta=\left(\gamma_{\mathrm{SV}}-\gamma_{\mathrm{SL}}\right) / \gamma_{\mathrm{LV}}$

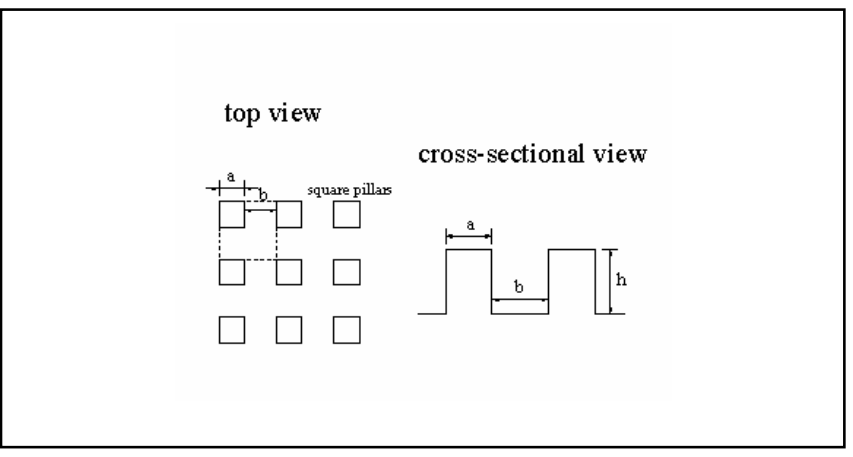

Figure 5. model of micro-structures on surface of PDMS replicas. 


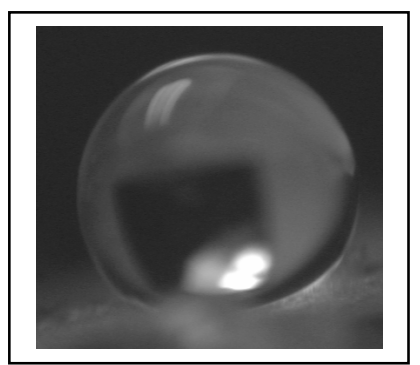

Figure 6. CA measurement on the PDMS replica sample $1, \mathrm{CA}=146^{\circ}$.

$\gamma$ is interfacial free energy per unit area, the subscripts SV, SL and LV represent solid-gas, solid-liquid and liquid-gas interface, respectively. Young's law can not be applied to a rough surface, Wenzel proposed a formula based on the hypothesis that liquids are in contact with the rough surface entirely:

$$
\cos \theta_{\mathrm{W}}=\mathrm{r} \cos \theta
$$

$\theta_{\mathrm{W}}$ is Wenzel type contact angle, $\mathrm{r}$ is the roughness factor, defined as the ratio of the actual area of a rough surface to the geometrically projected area. However, Cassie suggested that the droplet is only in contact with the top of the surface roughness, and is held upon the roughness. He proposed another formula:

$$
\cos \theta_{\mathrm{C}}=f \cos \theta+(1+f) \cos 180
$$

$\theta_{\mathrm{C}}$ is Cassie type contact angle, $f$ is the area fraction of wetted solid surface. There is an obvious difference between the contact angles defined by Wenzel's law and Cassie's law. Patankar [14] studied this difference, and pointed out that, if the droplet is in contact with the rough surface slightly without any extra energy, it would form Cassie type contact. If the droplet has enough energies, such as while dropping from some height, it will form Wenzel type contact. The contact can be transformed between Cassie type and Wenzel type, but the droplet should overcome the energy-barrier in the transformation.

He et al. [15] measured the contact angles on PDMS surfaces with different regular rough structures. The schematic diagram of these surface structures is shown in Fig. 5. They placed the droplets gently making them contact slightly or dropped them from some height to measure the Cassie type

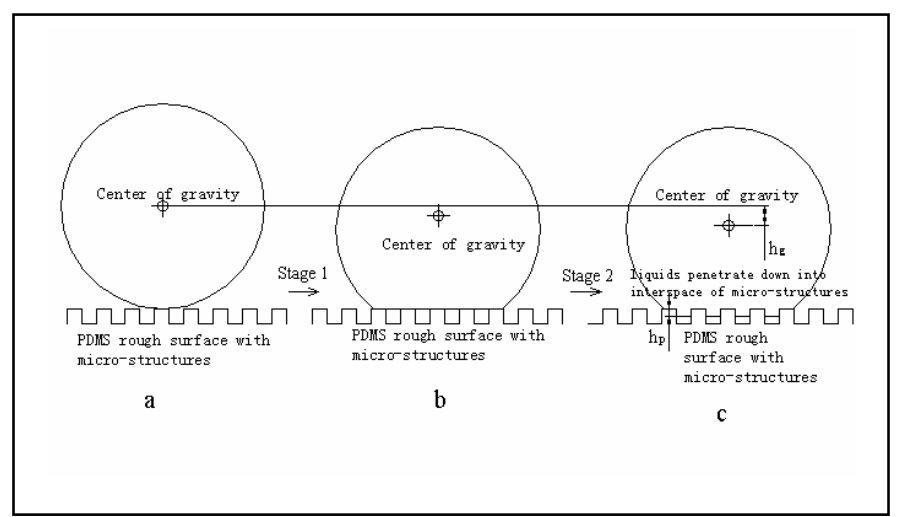

Figure 7. Schematic of liquids penetrating down into the interspace of micro-structures, $h_{P}$ is penetration height, and $h_{g}$ is the decreasing of gravity center of the droplet. contact angle or the Wenzel type one, respectively. Their results showed that the trend of contact angles contacting slightly on the rough surface is similar to the prediction of Cassie's law, and that of contact angles through dropping from some height to the rough surface is similar to the prediction of Wenzel's law. But the deviation from the theory prediction is also clear, this needs to be studied further.

\section{B. A revised formula}

In out experiments, the droplets are slightly in contact with the surface of PDMS replicas, so we obtained Cassie's contact angles. The experimental results show that contact angles are monotonically increasing with the interspace ratio $\mathrm{b} / \mathrm{a}$ in some range. The trend is consistent with the Cassie's law, but the values are different.

Wenzel's law assumes that the droplet is in contact with the rough surface entirely, but Cassie's law assumes that the droplet is held upon the top of the rough surface. The real state may be between these two conditions. Therefore we introduce a penetration height $h_{P}$, as shown in Fig. 7. The CA on rough surface $\theta^{\prime}$ can be defined as:

$\cos \theta^{\prime}=h_{\mathrm{P}} / \mathrm{h}\left(\cos \theta_{\mathrm{W}}\right)+\left(1-\mathrm{h}_{\mathrm{P}} / \mathrm{h}\right)\left(\cos \theta_{\mathrm{C}}\right)$

This formula is a weighted summation of Wenzel type CA $\theta_{\mathrm{W}}$ and Cassie type CA $\theta_{\mathrm{C}}$. The weight is $\mathrm{h}_{\mathrm{P}} / \mathrm{h}$, defined as the proportion of penetration height $h_{P}$ and height of rough structures $h$. The first term in the right-hand side of the formula is the contribution of liquids penetration down into the interspace among rough structures. The second term is the contribution of solid-liquid contact in the upper surface of the rough structures. Substituting (2), (3) into (4), we obtain:

$\cos \theta^{\prime}=\mathrm{h}_{\mathrm{P}} / \mathrm{h}(\mathrm{r} \cos \theta)+\left(1-\mathrm{h}_{\mathrm{P}} / \mathrm{h}\right)(f \cos \theta+f-1)$ where $f=a^{2} /(a+b)^{2}, \mathrm{r}=\left[(a+b)^{2}+4 a \mathrm{~h}\right] /(a+\mathrm{b})^{2}, \theta$ is the contact angle measured on a flat surface formed by the same solid material. Fig. 8 gives a comparison among Cassie's formula, Wenzel's formula and this revised formula. If $\mathrm{b}$ is much larger than $a$, then interspace ratio $\mathrm{b} / a$ tends to be infinite, $\mathrm{h}_{\mathrm{P}}$ will increase, so the limit of $\cos \theta^{\prime}$ is just $\cos \theta$. It means contact angle $\theta^{\prime}$ will tend to a contact angle in a flat surface $\theta$, it is certainly consistent with the real condition.

\section{Calculation of penetration height}

For calculating $\theta^{\prime}, h_{P}$ should be determined first. In an energy point of view, we may suppose a wetting process: the droplet (with radius $\mathrm{R}$ ) is in contact with the rough surface as shown in Fig. 7, and the surface micro-structures are the same as shown in Fig. 5. At the original state, just the lowest point of the droplet contacts to the surface, then the droplet will go down and form more liquid-solid contact areas. In this process, two stages may occur. One is liquids spread on the top of the micro-structures until the droplet base expands to an equilibrium state (Fig. 7b), and the other is liquids penetrate into the interspace between the micro-structures (Fig. 7c). So the center of gravity of the droplet finally decreases by a height $h_{C}$, and liquids penetrate down by a height $h_{P}$. Because the contact angles in our experiments are around $140^{\circ}$, the increase of droplet radius is just about $1 \%$, so the increase of surface area of the droplet due to deformation can be neglected. In the whole process, the gravity and the surface tension should be considered. The work done by gravity is:

$$
\mathrm{W}_{\mathrm{g}}=\mathrm{mgh}_{\mathrm{g}}
$$




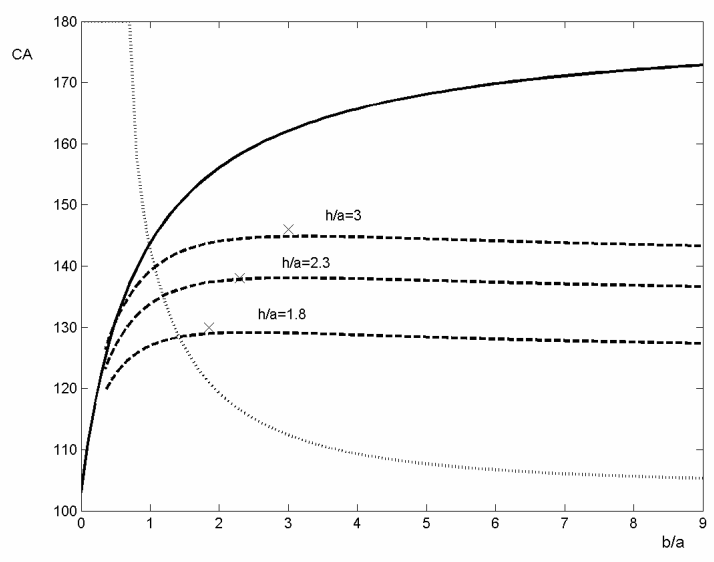

Figure 8. Comparison the experimental data of CA with the values of the results from Cassie's formula, Wenzel's formula and our revised formula. Solid line: Cassie's formula. Dotted line: Wenzel's formula.

Dashed line: revised formula (Eq. 5), h/a =1.8, 2.3 and 3, respectively. Crosses: Experimental results.

where $\mathrm{m}$ is the mass of the droplet, $\mathrm{g}$ is the gravity acceleration, $h_{g}$ is the decreasing of the gravity center of the droplet. The increase of surface free energy in stage one, with liquids spreading on the top of the micro-structures, is:

$\Delta \mathrm{G}_{1}=\mathrm{N}\left(\gamma_{\mathrm{SL}}-\gamma_{\mathrm{SV}}-\gamma_{\mathrm{LV}}\right) \mathrm{A}_{\mathrm{up}}$

where $\mathrm{A}_{\mathrm{up}}=a^{2}$, is the upper surface area of single micro-pillar, and $\mathrm{N}$ is the number of the micro-pillars in contact with the liquids. If the $\mathrm{CA}$ on the rough surface is measured as $\theta^{\prime}, \mathrm{N}$ can be estimated approximately as $\mathrm{N} \approx \pi\left(\operatorname{Rin} \theta^{\prime}\right)^{2} /(a+b)^{2}$. According to Young's formula (1), equation (7) can be simplified as:

$$
\Delta \mathrm{G}_{1}=-\mathrm{N} a^{2} \gamma_{\mathrm{LV}}(1+\cos \theta)<0
$$

The first stage will stop, when the horizontal forces reach an equilibrium state. Also, we may discover that, the system does not reach an energy steady state, liquids may penetrate down into the interspace between the micro-structures.

If the liquids penetrate down by $h_{P}$ in stage two, the increase of surface free energy is:

$$
\Delta \mathrm{G}_{2}=\mathrm{N}\left(\gamma_{\mathrm{SL}}-\gamma_{\mathrm{SV}}\right) \mathrm{A}_{\text {side }}
$$

where $\mathrm{A}_{\text {side }}=4 \mathrm{ah}$ is the side area of single micro-pillar in contact with liquids, $\mathrm{N}$ is the same as in equation (7). Substituting equation (1) into (9), we obtain:

$$
\Delta \mathrm{G}_{2}=-4 a \mathrm{~h}_{\mathrm{P}} \mathrm{N} \gamma_{\mathrm{LV}} \cos \theta
$$

Because in a flat hydrophobic surface CA $\theta>90^{\circ}$, so $\Delta \mathrm{G}_{2}>0$ in stage 2 , which means that liquids need extra energies to penetrate.

So the energy equilibrium equation is:

$\mathrm{Wg}=\Delta \mathrm{G}_{2}+\Delta \mathrm{G}_{2}$

Based on the study of Mahadevan and Pomeau [16], the decreasing of gravity center of droplet on flat surface $\mathrm{h}_{\mathrm{g}} \sim \mathrm{Bo} \cdot \mathrm{R}$, Bo is the Bond number, defined as $\mathrm{Bo}=\rho \mathrm{gR}^{2} / \gamma_{\mathrm{LV}}$, where $R$ and $\rho$ are radius and density of the droplet, respectively, $g$ is the gravity acceleration, $\gamma_{\mathrm{LV}}$ is the interfacial free energy per unit area on the liquid-gas interface. In our experiment, $\mathrm{Bo} \approx 0.05$, the work done by gravity is just about $3 \%$ of the total surface free energies, so gravity can be neglected. Substituting (6), (8) and (10) into (11), we obtain:

$$
a(1+\cos \theta)=4 \mathrm{~h}_{\mathrm{P}}(-\cos \theta)
$$

Substituting the value of $a$ and $\theta$ mentioned above into Equation (12), we could estimate that $h_{\mathrm{P}} \approx 3 \mu \mathrm{m}$.

With the calculated $h_{P}$, Fig. 8 shows the curves of revised formula (5). It gives the trend of $\theta^{\prime}$ with respect to the geometric sizes of surface micro-structures. The experimental results (crosses in Fig. 8) consist with the results of revised formula well. The CA has the largest values when $\mathrm{b} / a$ is about $2 \sim 4$, and there is a threshold value of interspace ratio $\mathrm{b} / a=2.5$, in which the largest CA can be achieved. So this revised formula (5) may serve as a criterion for designing the surface micro-structures.

\section{CONCLUSION}

This paper observed the micro/nano double-layer structures of the lotus leaves surfaces, studied the microstructures, and presented a simple description to these structures. To estimate the effects of the double-layer structures on surface hydrophobicity, we used PDMS to replicate the lotus leaves surfaces, and obtained surfaces with micro-structures of a real lotus leaf. The contact angles on these surface are from $130^{\circ}$ to $146^{\circ}$. Although this method can not replicate the nano-structures, the hydrophobicity of the "PDMS lotus leaf" is still very high. It means that the microstructures of surface play an important role in the hydrophobicity of surface in steady cases.

Based on Cassie's law and Wenzel's law, we reconsidered the wetting process when water contacts the surface with micro-structures, and proposed a revised formula to predict the contact angle on rough surface. With the revised formula, the largest $\mathrm{CA}$ can be obtained when $\mathrm{b} / a \approx 2 \sim 4$, and the CA reaches its maximum value when the interspace ratio $b / a \approx 2.5$.

It is worth researching the effects of the nano-structures on the lotus leaves surfaces. In an energy point of view, the nanostructures increase the surface area ratio, so that liquids have to wet more surface area when liquids penetrate down into the interspace. The higher surface area ratio leads to smaller value of penetration height $h_{P}$. From formula (5), we can obtain a larger contact angle. It needs further experiments on surface with nano-structures.

\section{ACKNOWLEDGMENT}

The authors gratefully acknowledge the discussion with the group of academician Gan Zi-Zhao in MEMS Center of Beijing University. We thank Mr. Xie Jijia and senior engineer Shen Huan for using SEM, and Dr.Cui for preparing PDMS replication. The support of this work by the Major Innovation Project of Chinese Academy of Sciences (KJCX2-SW-L2) and National Natural Science Foundation of China (10272107) are also gratefully acknowledged.

\section{REFERENCES}

[1] T. Nishino, M. Meguro, K. Nakamae, M. Matsushita, and Y. Ueda, "The lowest surface free energy based on $-\mathrm{CF}_{3}$ alignment," Langmuir, vol. 15 , pp.4321-4323, 1999.

[2] A. Nakajima, "Design of a transparent hydrophobic coating," Journal of the Ceramic Society of Japan, vol. 112(10), pp.533-540, 2004.

[3] W. Barthlott, C. Neinhuis, "Purity of the sacred lotus, or escape from contamination in biological surfaces," Planta, vol. 208, pp.1, 1997. 
[4] D.W. Bechert, M. Bruse, W. Hage, R. Meyer, "Fluid mechanics of biological surfacees and their technological application," Naturwissenschaften, vol. 87, pp.157-17, 2000

[5] R. Furstner, C. Neinhuis, W. Barthlott, "The lotus effect: Selfpurification of microstructured surfaces," NACHRICHTEN AUS DER CHEMIE, vol.48(1), pp.24-28, 2000.

[6] W. Barthlott, C. Neinhuis, "The lotus effect: A self-cleaning surface based on a model taken from nature," TEKSTIL, vol.50 (9), pp.461-465, 2001.

[7] X-F. Gao, L. Jiang, "Water-repellent legs of water striders," Nature, vol.432, pp.36, 2004.

[8] W. Lee, M-K, Jin, W-C, Yoo, J-K. Lee, "Nanostructuring of a polymeric substrate with well-defined nanometer-Scale topography and tailored surfacee wettability," Langmuir, vol.20, pp.7665-7669, 2004.

[9] G.S. Watson, J.A. Watson, "Natural nano-structures on insectspossible functions of ordered arrays characterized by atomic force microscopy," Applied surface science, vol.235, pp.139-144, 2004.
[10] D. Öner and T.J. McCarthy, "Ultrahydrophobic surfaces, effects of topography length scales on wettability," Langmuir, vol.16, pp.7777, 2000.

[11] T. Onda, S. Shibuichi, N. Satoh, K. Tsujii, "Super-Water-Repellent Fractal Surfaces," Langmuir, vol.12(9), pp.2125, 1996.

[12] N.A. Patankar, "Mimicking the lotus effect: influencee of double roughenss structures and slender pillars," Langmuir, vol.20, pp.8209$8213,2004$.

[13] J.C. Mcdonald, G.M. Whitesides, "Poly(dimethylsiloxane) as a materiall for fabricating microfluidic devices," Accounts of chemical research, vol.35(7), pp.491, 2002.

[14] N.A. Patankar, "Transition between superhydrophobic states on rough surfaces," Langmuir, vol.20, pp.7097-7102, 2004.

[15] B.He, N.A. Patankar, J. Lee, "Multiply equilibrium droplet shapes and design criterion for rough hydrophobic surfaces," Langmuir, vol.19, pp.4999, 2003.

[16] L. Mahadevan, Y. Pomeau, "Rolling droplets," Physics of fluids, vol.11(9), pp.2449-2453, 1999. 